\title{
Analyzing and Projection of Future Bangladesh Population Using Logistic Growth Model
}

\author{
Mohammad Sharif Ullah'1 (1), Golam Mostafa², Nusrat Jahan³ ${ }^{3}$ Md. Abdul Hakim Khan ${ }^{4}$ \\ ${ }^{1}$ Department of Mathematics, FENI UNIVERSITY, Feni, Bangladesh \\ ${ }^{2}$ Department of Mathematics, Hamdard University Bangladesh, Munshiganj, Bangladesh \\ ${ }^{3}$ Department of Mathematics, Bangladesh Army International University of Science \& Technology, Comilla, Bangladesh \\ ${ }^{4}$ Department of Mathematics, Bangladesh University of Engineering \& Technology (BUET), Dhaka, Bangladesh \\ Email: sharifju49@yahoo.com, mostafamath02@gmail.com, nusrat.seeme@gmail.com,mahkhan@math.buet.ac.bd
}

How to cite this paper: UllaH, M.S., Mostafa, G., Jahan, N. and Khan, Md.A.H. (2019) Analyzing and Projection of Future Bangladesh Population Using Logistic Growth Model. International Journal of Modern Nonlinear Theory and Application, 8, 53-61. https://doi.org/10.4236/ijmnta.2019.83004

Received: January 8, 2019

Accepted: July 2, 2019

Published: July 5, 2019

Copyright (c) 2019 by author(s) and Scientific Research Publishing Inc. This work is licensed under the Creative Commons Attribution International License (CC BY 4.0).

http://creativecommons.org/licenses/by/4.0/

\begin{abstract}
Bangladesh is a densely populated country than many other countries of the world. The population growth is termed as alarming, however, knowledge of growth in the years to come would be useful in planning for the development of the country. This article is based on the projection of future population growth of the country. The available actual population census data during 1991-2011 of Bangladesh was applied to the application of a non-linear, non-autonomous ordinary differential equation familiar as Verhulst logistic population model with the maximum environmental capability of Bangladesh. Bangladesh will reach its carrying capacity of 245.09 million population in the next 56 years i.e. the year 2067 and then it decreases as S-shaped curve. The article has provided a focus on the changing trends of the growth of the population of Bangladesh.
\end{abstract}

\section{Keywords}

Logistic Model, Carrying Capacity, Zero Growth Rate, Least Square Method, $6^{\text {th }}$ Order RK Method, Future Population Projection, Parabolic Profile

\section{Introduction}

Bangladesh is a small country with a huge ( $8^{\text {th }}$ most in the world) population in South Asia. Overpopulation is the main cause of the poverty, illiteracy, unemployment, and malnutrition of the country. So the aggregative tendency in population growth is a great alarming to the people of Bangladesh. Therefore population control should be given top most priority in planning national policy. Application and use of a feasible technique of estimation and projection of the 
future population of the country can find importance at this juncture. Population dynamics uses population model, basically leading an individual form of a mathematical model. There are a lot of techniques which assist to develop a sound population model. Among these, Malthusian exponential model by Thomas Robert Malthus (1766-1834) and the Verhulst (1838) logistic differential equation model are well known [1]. The exponential model cannot define environmental limit point as it provides a stress-free calculation for future population and cannot catch the meticulous future population number. So the population will increase with the phase of time to infinity which is unrealistic. Main limitation of Malthus model is that the population is increasing geometrically and twice in 25 or more years [2]. This model is appropriate for a very small time and a small amount of population as it doesn't reflect the environmental dynamics. The Logistic model is the adjustment of Malthus model. The logistic model states the growth of population which decreases the population while reaching the carrying capacity level. It theorizes the surplus of birth over death in per unit time and doesn't enumerate for the drawbacks of resources. After reaching maximum level of population size, it decreases like S-shaped curve. In comparison, the exponential model doesn't provide estimation more accurate than a logistic one.

Population census data of Bangladesh from 1991 to 2011 were used to arrive at an accurate prediction of the population. The growth rate in the population of Bangladesh has been strictly decreasing, its overall population has been strictly increasing and it would continue to increase until a zero growth rate is reached. The country will have the largest population when the growth rate will be zero. The logistic model is found to be used in census data of different country populations [3] [4]. Obaidullah (1976) presented an "Expo-linear Model" claiming that this model is superior to exponential or linear model in unfolding population growth over time [5]. However, there can be a difficulty in understanding its basic parameter unlike that of exponential or linear one. Mallik (1980) studies the motion of population in Bangladesh and suggested the prospect of a zero population growth rate in the ensuing hundred years (2080) [6]. Beekman (1981) had modified exponential growth model. That model had been applied a Markov chain to reproduce lower birth rate instigated by rural-urban development [7]. Applying this model, he estimated the future population of Bangladesh in 1998, 2018 and 2038 correspondingly. Kabir \& Chowdhury (1982) scrutinized the affiliation between the population growth and food production of Bangladesh [8]. They suggested that the government should take necessary steps such as feeding policy etc. for declining the alarming growth of population in Bangladesh. Rabbani \& Shadat Hossain (1981) studied the population trend of Bangladesh and forecasted the population of Bangladesh from 1975 to 2025 which is considered as the foundation work of forecasting population of Bangladesh [9]. The main limitation of their work is that they cannot indicate the year when Bangladesh will reach the apex of population. Though some researchers forecast 
on the population of Bangladesh, someone has used data of world meter or UN which is petite different from Bangladesh actual census data. But we carry on our work using actual census data of population of Bangladesh.

At the backdrop of stringent economy and environment of the country, it is crucially important to comprehend the fluctuating population movement for better planning in the country, in the days to come. The principal aim of the exercise was to prepare an estimation of growth of Bangladesh by using logistic model in predate actual census data from the year 2012 onward, up to 1991 to 2011. The Logistic model used and applied in population data (Table 1) was found to work accurately in the estimation of population of Bangladesh and thus it is hoped that the findings will help in successful population projection, planning and development of the country, in next future.

\section{Methodology}

The method of arriving at reliant elucidations to the problems through the methodical assortment, analyzing and explanation of data was one of the bestunspoken tools in any kind of research work. For this research work, secondary classified census data of Bangladesh (1991-2011) were collected from the Bangladesh Bureau of Statistics, the government of Bangladesh. $6^{\text {th }}$ order RK method ( $6^{\text {th }}$ order) in logistic model through MATLAB programming was used for the direct projection of future population of Bangladesh. Verhulst deduced formula was used to find out the carrying capacity and growth rate of Bangladesh population. Least square interpolation method was used to enumerate the growth rate of the future population of Bangladesh as a function of time.

\section{Mathematical Model}

A mathematical model is a benign picture of the whole structure using mathematical thoughts and verbal.

\subsection{Malthusian Exponential Growth Model}

Malthusian Growth Model, sometimes entitled a simple exponential growth model, is essentially exponential growth based on a constant rate. The model is named after Thomas Robert Malthus, who wrote "An Essay on the Principle of Population" (1798), one of the most primitive and prominent books on population dynamics [2]. It reflects exponential growth of population and can be termed by the first order linear differential equation as the form:

Table 1. Census data of Bangladesh [10].

\begin{tabular}{cc}
\hline Year & Bangladesh Census Data (in thousand) \\
\hline 1991 & 106,313 \\
2001 & 124,355 \\
2011 & 142,319
\end{tabular}




$$
\frac{\mathrm{d} N}{\mathrm{~d} t}=r N
$$

where $r$ is the growth rate (Malthusian Parameter).

Equation (1) is habitually mentioned as the exponential law. It is usually considered in the arena of population ecology as the leading principle of population dynamics where Malthus as the initiator. Also occasionally it mentioned as the Malthusian Law.

The solution to Equation (1) is easily achieved by separation of variables and integrating both sides of the equation, supposing that $N(0)=N(t)=0$, to yield

$$
\int_{N(0)}^{N(T)} \frac{\mathrm{d} N}{N}=\int_{t=0}^{t=T} r \mathrm{~d} t \Rightarrow \ln |N|_{N(0)}^{N(T)}=\left.r t\right|_{0} ^{T}+C
$$

and evaluating the upper and lower limits yields

$$
\begin{aligned}
& \ln (N(T))-\ln (N(0))=(r T+C)-(r \times 0+C) \\
& \Rightarrow \frac{N(T)}{N(0)}=\mathrm{e}^{r T}
\end{aligned}
$$

where $N(T)$ and $N(0)$ are both positive.

Rearranging the equation, we get the exact solution

$$
N(t)=N_{0} \mathrm{e}^{r t}
$$

where the initial condition $N(0)=N_{0}$.

\subsection{Logistic Model}

One of the utmost elementary and revolutionary models of population growth was the logistic model of population growth articulated by Pierre François Verhulst in 1838 [3]. The logistic model takes the shape of a sigmoid curve and describes the growth of population as exponential, followed by a reduction in growth, and bound by a carrying capacity due to ecological stresses which can be expressed first-order non-linear differential equation as the form:

$$
\frac{\mathrm{d} N}{\mathrm{~d} t}=r N\left(1-\frac{N}{K}\right)
$$

where parameter $r$ is the growth rate and $K$ is environmental maximum support i.e. limiting population as $t \rightarrow \infty$ and $N$ is the population size.

Solving Equation (3) for $N$, talking integration on both sides we get,

$$
\ln \left|\frac{K}{N}-1\right|=-r t+c \Rightarrow \frac{K}{N}-1=\mathrm{e}^{-r t+c} \Rightarrow \frac{K}{N}-1=A \mathrm{e}^{-r t}
$$

where $A=\mathrm{e}^{c}=$ constant .

Assuming $N=N_{0}$ (initial population) at $t=0$ then we get, $A=\frac{K}{N_{0}}-1$

$$
\therefore N=\frac{K}{1+A \mathrm{e}^{-r t}}=\frac{K}{1+\left(\frac{K}{N_{0}}-1\right) \mathrm{e}^{-r t}}
$$


Taking limit as $t \rightarrow \infty$ (since $r>0$ ), then from Equation (4) we get,

$$
N_{\text {max }}=K
$$

Suppose that at time $t=1$ and $t=2$ the values of $N$ are $N_{1}$ and $N_{2}$ respectively.

Then from Equation (4) we get,

$$
\frac{1-\mathrm{e}^{-r}}{K}=\frac{1}{N_{1}}-\frac{\mathrm{e}^{-r}}{N_{0}}, \frac{1-\mathrm{e}^{-2 r}}{K}=\frac{1}{N_{2}}-\frac{\mathrm{e}^{-2 r}}{N_{0}}
$$

Dividing $2^{\text {nd }}$ portion of Equation (6) by $1^{\text {st }}$ portion of Equation (6) we get,

$$
\begin{aligned}
& 1+\mathrm{e}^{-r}= \frac{\frac{1}{N_{2}}-\frac{\mathrm{e}^{-2 r}}{N_{0}}}{\frac{1}{N_{1}}-\frac{\mathrm{e}^{-r}}{N_{0}}} \\
& \Rightarrow \mathrm{e}^{-r}=\frac{N_{0}\left(N_{2}-N_{1}\right)}{N_{2}\left(N_{1}-N_{0}\right)}
\end{aligned}
$$

Putting the value of $\mathrm{e}^{-r}$ in first portion of Equation (6) and rearranging we get,

$$
K=\frac{N_{0} N_{1}^{2}+N_{1}^{2} N_{2}-2 N_{0} N_{1} N_{2}}{N_{1}^{2}-N_{0} N_{2}}
$$

which is the limiting value of $N$.

Let, at time $t=T$ population be $N=N_{1}$, where $T$ is equally spaced years.

Then form Equation (4) we can write,

$$
\begin{aligned}
& N_{1}=\frac{K}{1+\left(\frac{K}{N_{0}}-1\right) \mathrm{e}^{-r T}} \\
& \Rightarrow 1+\left(\frac{K}{N_{0}}-1\right) \mathrm{e}^{-r T}=\frac{K}{N_{1}} \\
& \Rightarrow\left(\frac{K}{N_{0}}-1\right) \mathrm{e}^{-r T}=\frac{K}{N_{1}}-1 \\
& \Rightarrow \mathrm{e}^{-r T}=\frac{\frac{K}{N_{1}}-1}{\frac{K}{N_{0}}-1} \\
& \Rightarrow \mathrm{e}^{r T}=\frac{\frac{K}{N_{0}}-1}{\frac{K}{N_{1}}-1} \\
& \Rightarrow r=\frac{1}{T} \ln \left(\frac{\frac{K}{N_{0}}}{\frac{K}{N_{1}}-1}\right)
\end{aligned}
$$




$$
\Rightarrow r=\frac{1}{T} \ln \left(\frac{\frac{1}{N_{0}}-\frac{1}{K}}{\frac{1}{N_{1}}-\frac{1}{K}}\right)
$$

\section{Determination of Carrying Capacity and Growth Rate}

According to Pierre François Verhulst we calculate the parameters $K$ and $r$ from the population $N(t)$ in three dissimilar consecutive years [3]. If $N_{0}$ is the population at time $t=0, N_{1}$ at time $t=T$ and $N_{2}$ at time $t=2 T$, then a monotonous calculation beginning from the exact solution of logistic model shows that Equation (8) and (9). For the population of Bangladesh in the years 1991, 2001 and 2011 correspondingly 106,313,000, 124,355,000 and 142,319,000, we obtained $K=245096511$ and $r=0.02960160617$ approximately.

Proceeding this way, we get Table 2.

Percentage of relative error of exponential model in 1991 is $0 \%$, in 2001 is $11.394 \%$ and 2011 is $35.033 \%$ while Percentage of relative error of logistic model in 1991 is $0 \%$, in 2001 is $0.002 \%$ and 2011 is $0.004 \%$. The error term of logistic has little difference to the census data of the population of Bangladesh. From Table 2, we can state that our logistic model data for the same period of time has matched as congenial harmony with the census data of the population of Bangladesh.

\section{Estimation of Future Population of Bangladesh Using Logistic Model}

From Equation (3) we can write,

$$
\frac{\mathrm{d} N}{\mathrm{~d} t}=r(t) N(t)\left(1-\frac{N(t)}{K}\right)
$$

where $K$ is assumed to be constant which is determined by the formula of Equation (8)

$$
\therefore(10) \Rightarrow \frac{1}{N(t)} \frac{\mathrm{d} N}{\mathrm{~d} t}=r(t)\left(1-\frac{N(t)}{K}\right)=F(t)
$$

future growth rate of Bangladesh population.

Table 2. Census, exponential and logistic model data.

\begin{tabular}{cccccc}
\hline Year & $\begin{array}{c}\text { Actual Census } \\
\text { Data of Bangladesh }\end{array}$ & $\begin{array}{c}{ }^{1} \text { Population } \\
\text { by exponential model }\end{array}$ & ${ }^{1}$ Error & $\begin{array}{c}{ }^{2} \text { Population } \\
\text { by logistic model }\end{array}{ }^{2}$ Error \\
\hline 1991 & $106,313,000$ & $106,313,000$ & 0.0 & $106,313,000$ & 0.0 \\
2001 & $124,355,000$ & $142,936,945.14$ & $14,169,339.51$ & $124,355,034$ & 34 \\
2011 & $142,319,000$ & $192,177,535.08$ & $49,858,535.08$ & $142,319,066.3$ & 66.3 \\
\hline
\end{tabular}




$$
\Rightarrow r(t)=\frac{F(t)}{1-\frac{N(t)}{K}}
$$

where $N(t)<K$ (carrying capacity)

Estimating $r(t)$, we model $F(t)$ with a linear equation in time.

So,

$$
F(t)=F(0)+m t
$$

where $m=\frac{a(2011)-a(2001)}{10}$ is determined by the given data in Table 1 .

According to least square interpolation, $F(t)=0.26554935849191336-0.029621318688351928 t \quad[11]$.

Now applying Equations (4) \& (12) with $6^{\text {th }}$ order RK method and MATLAB programming we estimate future Bangladesh population.

\section{Result \& Discussion}

We predicted the population of Bangladesh applying Verhulst logistic population model. Firstly we have analyzed the census data of population of Bangladesh during 1991-2011 compared with the logistic model that matched as congenial harmony with the census data of population of Bangladesh. To make the non-linear differential equation as linear we used least square interpolation method and expressed growth rate $F(t)$ as a function of $t$. Mathematically, the tactic of logistic model is a congenial apparatus to forecast the population of any country like Bangladesh. Higher order Runge-Kutta method is more accurate than lower order because of less error. Using higher order RK method ( $6^{\text {th }}$ order) in logistic model through MATLAB programming we may assume the future population of Bangladesh. For prediction, we use a generalized logistic population model. Figure 1 indicates that the graph of the forecasting population values is a sigmoid curve. Figure 2 states that the population of Bangladesh will gradually decrease in a parabolic manner i.e. sigmoid curve. This shows that the predicted values are fitted with the logistic model curve. Figure 1 indicates that Bangladesh to reach its carrying capacity would take more than 56 years i.e. the year of 2067. After 2067 growth rate will be zero and Bangladesh have the largest population and largest population will be 245.09 million. Due to the rapidly changing population, various natural disasters may occur as the population exceeds maximum population level i.e. environmental carrying capacity. Bangladesh government should take preventive measures and accelerates planning for "worst-case" outcome. This study introduces a vital role for better sustainable development plans with limited resources through the accurate idea of future population \& related information of resources. As future would follow the earlier, a projection has established on the basis of earlier trends and affiliations which nurture our apprehensions of the dynamics of population growth and often provide the change of future population trends that adequately minute to assist respectable assessment. Projection of future population gives a future 


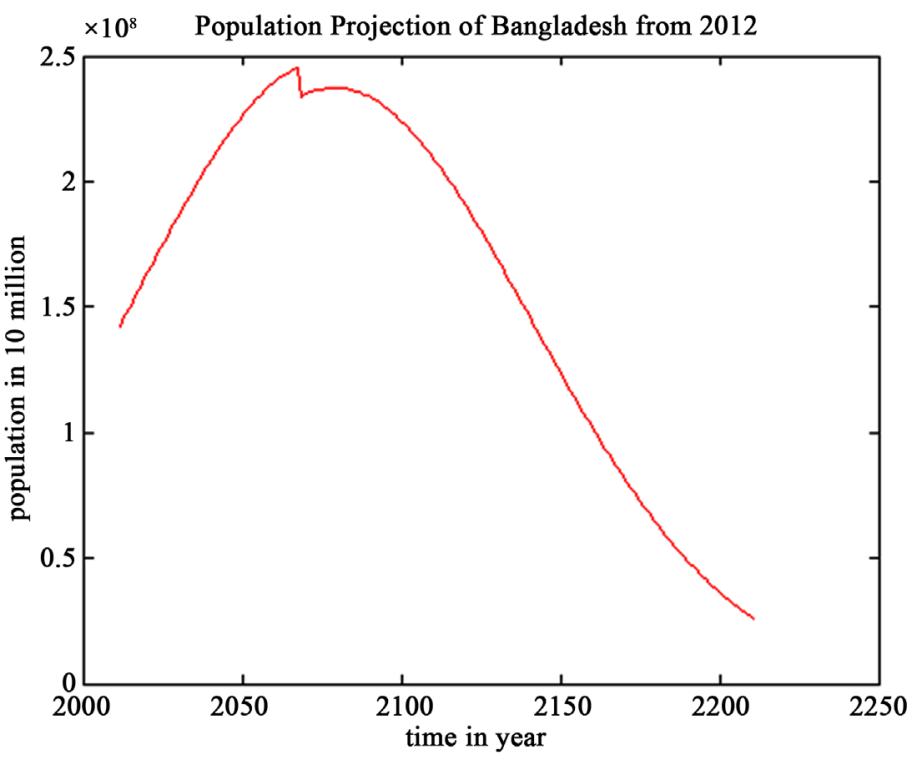

Figure 1. Projection curve of Bangladesh population.

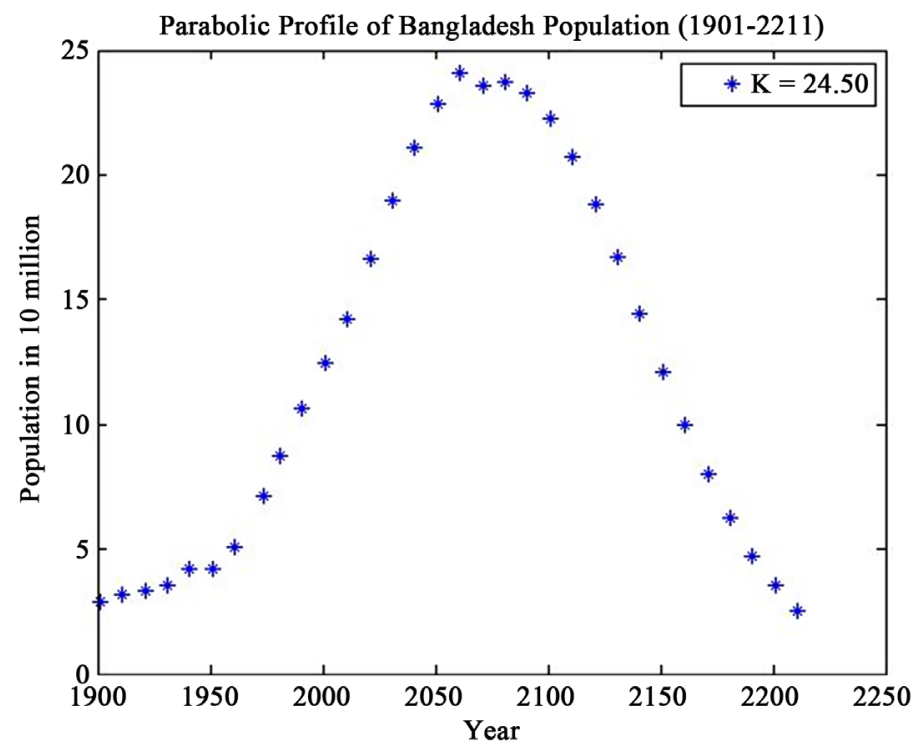

Figure 2. Parabolic profile of Bangladesh population (Census 1901-2011 and projected data 2012-2211).

portrait of the population which is well-disciplined by reducing population growth with different likely measures.

\section{Conclusion}

Finally, it was obvious that the methodology of logistic model had amalgamated with $6^{\text {th }}$ order RK method which was a congenial apparatus in population estimation. The principal aim of the exercise was to prepare an estimation of growth of Bangladesh by using logistic model in predate data from the year 2012 onwards, up to 1991 to 2011. The realistic mathematical logistic model is convenient in many disciplines from experimental utensils to arduous mathematical 
study and methods. Hence, the findings have been taken from this research work which will assist the government of Bangladesh in successful population planning and sustainable development in the country.

\section{Conflicts of Interest}

The authors declare no conflicts of interest regarding the publication of this paper.

\section{References}

[1] Murray, J.D. (2002) Mathematical Biology: An Introduction. Springer, New York, 1-75. https://doi.org/10.1007/b98868

[2] Malthus, T.R. (1798) An Essay on the Principle of Population, Introduction by Philip Appleman, and Assorted Commentary on Malthus Edited by Appleman: Library of Economics. Norton Critical Editions, ISBN 0-393-09202-X.

[3] Verhulst, Pierre-François (1845) Recherche s mathématiques sur la loi d'accroissement de la population Mathematical Researches into the Law of Population Growth Increase. Nouveaux Mémoires de 1 Académie Royale des Sciences et BellesLettres de Bruxelles, 18, 1-42.

[4] Raymond Pearl and Reed, L.J. (1920) On the Rate of Growth of the Population of the USA Since 1790 and Its Mathematical Representation. Proceeding of the National Academy of Science, 6, 275-288. https://doi.org/10.1073/pnas.6.6.275

[5] Obaidullah, M. (1976) Expo-Linear Model for Population Growth. Rural Demography, 3, 43-69.

[6] Mallick, A.A. (1980) Implausibility of Attaining Zero Population Growth in Banthin Next 100 Years. Rural Demography, 7, 33-39.

[7] Beekman, J.A. (1981) Several Demographic Projection Techniques. Rural Demography, 8, 1-11.

[8] Kabir, M. and Chowdhury, A.A. (1982) Population Growth and Food Production in Bangladesh. Rural Demography, 9, 25-56.

[9] Ghulam Rabbani, A.K.M. and Md Shadat Hossain (1981) Population Projection of Bangladesh (1975-2025), BBS, Dhaka.

[10] BBS, Bangladesh Population Census-2011, Bangladesh Bureau of Statistics, Government of Bangladesh, Dhaka, 2011.

[11] Mathews, J.H. (1992) Bounded Population: A curve Fitting Lesson. Mathematics and Computer Science, 26, 2-31.

\section{Nomenclature}

$t$. Time $t$ in years

$N_{t}$ : Population at time $t$

$N_{0}$ : Population at reference time $t=0$ i.e. year 1991

$K$ : Human population capacity i.e. carrying capacity

$r$. Growth rate

$\frac{\mathrm{d} N}{\mathrm{~d} t}$ : Rate of change of population with respect to time $t$

$F(t)$ : Future growth rate of Bangladesh Population 